\title{
Shared Molecular Mechanisms in Alzheimer's Disease and Amyotrophic Lateral Sclerosis: Neurofilament-Dependent Transport of SAPP, FUS, TDP-43 and SOD1, with Endoplasmic Reticulum-Like Tubules
}

\author{
Virgil Muresan Zoia Ladescu Muresan \\ Department of Pharmacology, Physiology and Neuroscience, New Jersey Medical School, Rutgers, \\ The State University of New Jersey, Newark, N.J., USA
}

\section{Key Words}

Alzheimer's disease - Amyotrophic lateral sclerosis .

Amyloid- $\beta$ precursor protein - FUS · TDP-43 - SOD1 .

Motor neurons - Neurofilaments - Endoplasmic reticulum .

Converging mechanisms of neurodegenerative diseases

\begin{abstract}
Background: Amyotrophic lateral sclerosis (ALS), a debilitating neurodegenerative disorder of the motor neurons, leads to the disorganization of the neurofilament (NF) cytoskeleton and - ultimately - the deterioration of the neuromuscular junction. Some familial cases of ALS are caused by mutated FUS, TDP-43 or SOD1; it is thought that the mutated proteins inflict pathology either by gain or loss of function. The proper function of the neuromuscular junction requires SAPP, a soluble proteolytic fragment of the amyloid- $\beta$ precursor protein (APP) - a transmembrane protein implicated in the pathology of Alzheimer's disease (AD). Whether sAPP, FUS, TDP-43 and SOD1 are mechanistically linked in a common pathway deregulated in both AD and ALS is not known. Summary: We show that SAPP, TDP-43, FUS and SOD1 are transported to neurite terminals by a mechanism that involves endoplasmic reticulum (ER)-like tubules and requires peripherin NFs. The transport of these proteins, and the
\end{abstract}

translocation of the ER protein reticulon 4 (Rtn4) into neurites was studied in CAD cells, a brainstem-derived neuronal cell line highly relevant to AD and ALS. We show that a significant fraction of SAPP is generated in the soma and accumulates in a juxtanuclear ER subdomain. In neurites, sAPP localizes to Rtn4-positive ER-like tubules that extend from the soma into the growth cone and colocalizes with peripherin NFs. Knocking down peripherin disrupts the NF network and diminishes the accumulation of SAPP, TDP-43, FUS, SOD1 and Rtn4 at terminals. Key Messages: We propose that the impediment of a common, ER-mediated mechanism of transport of SAPP, TDP-43, FUS and SOD1, caused by a disrupted NF network, could be part of the mechanisms leading to $A D$ and $A L S$.

(c) 2015 S. Karger AG, Basel

\section{Introduction}

An emerging concept in the field of neurodegenerative disease research is that many of these diseases display various degrees of overlapping pathology. Moreover, proteins linked to pathogenesis in one disease could also be implicated in other, apparently unrelated diseases. This suggests that the molecular mechanisms operating in dis-

\section{KARGER}

E-Mail karger@karger.com

www.karger.com/ndd
(C) 2015 S. Karger AG, Basel

$1660-2854 / 15 / 0162-0055 \$ 39.50 / 0$
Dr. Zoia Ladescu Muresan

Department of Pharmacology, Physiology and Neuroscience New Jersey Medical School, Rutgers, The State University of New Jersey 185 South Orange Avenue, MSB, I-665, Newark, NJ 07101-1709 (USA)

E-Mail muresazo@njms.rutgers.edu 
tinct neurodegenerative diseases could share common components, from single proteins to entire signaling cascades or cellular functions. A case in point is that of Alzheimer's disease (AD) and amyotrophic lateral sclerosis (ALS), two unrelated neurodegenerative diseases that primarily target either the central nervous system (in $\mathrm{AD}$ ) or the peripheral nervous system (in ALS). Both diseases are largely sporadic, meaning that they have no precisely identified genetic cause, although susceptibility genes exist. However, the familial types - which are inherited point to genes that, when mutated, lead unavoidably to disease. One such gene codes for amyloid- $\beta$ precursor protein (APP); certain mutations in the APP gene cause early-onset AD. The situation is similar in ALS, where a number of genes cause the disease, when mutated; among these are SOD1, FUS, TARDBP (encoding TDP-43), $P R P H$ (encoding peripherin, a neurofilament, NF, protein). Interestingly, while only a small fraction of the familial cases show mutations in genes encoding NF proteins, virtually all cases of ALS are afflicted by a disorganized NF cytoskeleton [1].

A recent study proposed that $\mathrm{APP}$, an $\mathrm{AD}$-linked gene product, actively contributes to the neuronal pathology of certain forms of ALS [2]. Also, APP was proposed to be essential for the proper function of the neuromuscular junction, the primary target of ALS pathology (reviewed in Caldwell et al. [3]). Conversely, the abnormal expression and aggregation of the ALS-linked gene product, TDP-43, a DNA/RNA-binding protein, was reported in some $\mathrm{AD}$ cases $[4,5]$, while SOD1 clearly regulates the APP metabolism $[6,7]$, raising the possibility of converging mechanisms leading to disease in AD and ALS.

Here, we provide evidence that proteins linked to $A D$ and ALS could use a common, yet unusual, form of axonal transport that relies on endoplasmic reticulum (ER)like tubules as vehicle for transport and is regulated by NFs. We propose that this transport mechanism, carrying soluble APP (sAPP; or other N-terminal fragments of APP), as well as FUS, TDP-43 and SOD1 to the synaptic terminal, could become disrupted and lead to disease. These results extend our previous studies pointing to overlapping mechanisms of disease in AD and ALS [8].

\section{Methods}

The neuronal cell line CAD [9], extensively employed by us in AD-relevant work [10-12], was used throughout these studies. CAD neuronal cells are derived from the brainstem, a region that is afflicted with neuronal pathology both in AD and ALS. Similar to the motor neurons, they express all major NF proteins, includ- ing peripherin, as well as proteins relevant to ALS: FUS, TDP-43, SOD1. CAD neuronal cells also express APP (the 695-amino-acid, neuronal isoform), which is cleaved by secretases to generate the characteristic, $\mathrm{AD}$-relevant fragments: $\mathrm{sAPP} \beta, \mathrm{C}$-terminal fragment- $\beta$ (CTF $\beta)$, amyloid- $\beta$ peptide and APP intracellular domain [13-15]. Immunoblotting, immunocytochemistry and transfection with dual-tagged APP (FLAG-APP-Myc) [16], or peripherin siRNA (Santa Cruz Biotechnology) plus green fluorescent protein (Lonza; to visualize transfected cells), were done as described [17, 18]. The following antibodies were used: anti-APP N-terminal region (22C11; Millipore), anti-APP C-terminal region (Y188; Epitomics), anti-SOD1 (Santa Cruz), anti-FUS/TLS, and anti-TDP-43 (Proteintech). As previously shown, in CAD neuronal cells, the antibody 22C11 largely detects SAPP, rather than full-length APP $[8,13,16]$. To retest specificity of labeling, antibody $22 \mathrm{C} 11$ was preadsorbed on a membrane that contained the APP region of an overloaded transfer of rat brain lysate (similar to the blot in fig. $2 \mathrm{D}$ in Muresan et al. [13]) to remove the anti-APP immunoreactive species from the IgG fraction, or preadsorbed on a membrane that contained transferred BSA. The antibody preadsorbed on APP shows no labeling in immunocytochemistry of CAD cells (fig. 1d). The anti-peripherin and one of the anti-APP C-terminal region (C9) antibodies were kindly provided by Dr. Robert Goldman (Northwestern University) and Dr. Denis Selkoe (Harvard Medical School), respectively. An additional anti-peripherin antibody (clone 8G2) was from Sigma. Anti-reticulon-4 (Rtn4) antibodies were either from LSBio or kindly provided by Dr. Riqiang Yan (Cleveland Clinic Foundation). In some experiments, CAD cells were incubated with $10 \mu \mathrm{g} / \mathrm{ml}$ brefeldin A (BFA; Sigma) for either 22 or $1.5 \mathrm{~h}$; in the latter case, a 1.5-hour washout period followed, prior to fixing the cells. For immunocytochemistry experiments, digital images were acquired with an Olympus IX81 microscope equipped with Semrock filters, cooled CCD camera (Hamamatsu Photonics) and Image-Pro Plus software (Media Cybernetics). Box plots were generated with BoxPlotR, a web-tool of the Tyers (http://tyers.iric.ca/) and Rappsilber (http://rappsilberlab.org/) laboratories [19].

\section{Results}

Using brainstem-derived CAD neuronal cells and primary neurons in culture, we have shown that a significant fraction of APP is cleaved in the neuronal soma, and that the generated sAPP segregates from the CTF $[13,16]$ (as revealed by immunocytochemistry with antibodies to epitopes in the $\mathrm{N}$ - and C-terminal regions of APP, referred to as $\mathrm{APP}_{\mathrm{N}}$ and $\mathrm{APP}_{\mathrm{C}}$, respectively; see online suppl. fig. 1a; for all online suppl. material, see www. karger.com/doi/10.1159/000439256) and accumulates in a cell compartment colocalized with the perinuclear peripherin NFs [8]. The identity of this compartment, and the mechanism of sAPP transport to the neurite terminals, is unknown. We now show that, in the soma, sAPP is enriched in an ER subdomain within the juxtanuclear region, where it largely colocalizes with Rtn4, a trans- 

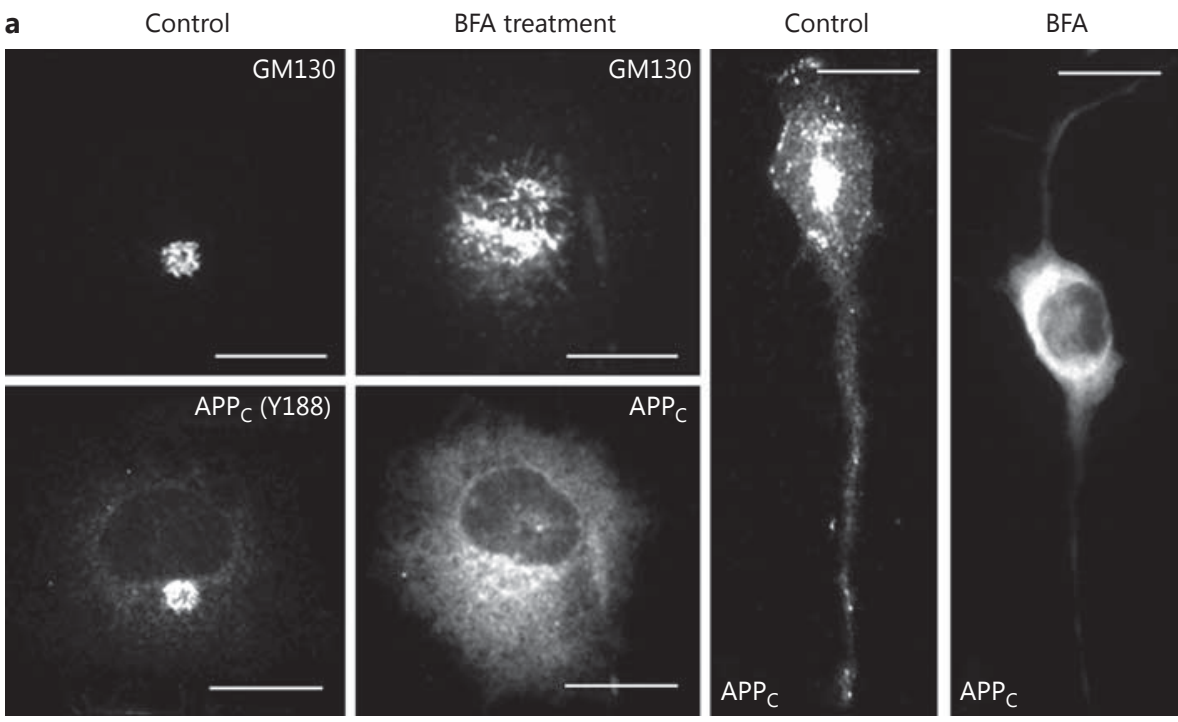

Fig. 1. a, b Transport into neurites of sAPP is not affected by brefeldin A (BFA) treatment. CAD cells were subjected to BFA treatment for $22 \mathrm{~h}$ and probed for the distribution of GM130 (a Golgi marker), APP C-terminal $\left(\mathrm{APP}_{\mathrm{C}}\right.$, detected with antibody $\mathrm{Y} 188 ; \mathbf{a})$ and $\mathrm{N}$-terminal $\left(\mathrm{APP}_{\mathrm{N}}\right.$, detected with antibody $22 \mathrm{C} 11$; b) epitopes, and Rtn4 (b). Controls without BFA treatment are also shown. c The box plot shows that BFA treatment blocks translocation of phosphorylated $\mathrm{APP}_{\mathrm{C}}\left(\mathrm{pAPP}_{\mathrm{C}}\right)$, but not of $\mathrm{APP}_{\mathrm{N}}$, into neurites. Translocation of nonphosphorylated $\mathrm{APP}_{\mathrm{C}}$ is also blocked by BFA treatment. d Control, dual labeling experiment, showing specificity of the $22 \mathrm{C} 11$ antibody for APP species. Antibody 22C11 was used after preadsorption on APP, a procedure that removed the anti-APP immunoreactive species from the IgG fraction (see Methods). Preadsorption on BSA revealed the typical immunolabeling with antibody 22C11, similar to that seen in $\mathbf{b}$ (not shown). The blot on the right shows that antibody $22 \mathrm{C} 11$ detects polypeptides in the region of APP/sAPP $(*)$. In a previous study, we showed that $\mathrm{SAPP} \beta$ migrates within this region of the blot and represents a substantial fraction of the immunolabeled band [16]. Molecular weight markers are given in kilodaltons. Bars $=20 \mu \mathrm{m}$ (a, b) and $50 \mu \mathrm{m}(\mathbf{d})$.
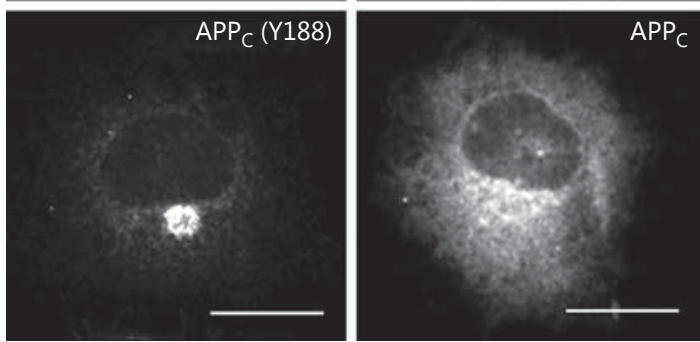

b Control
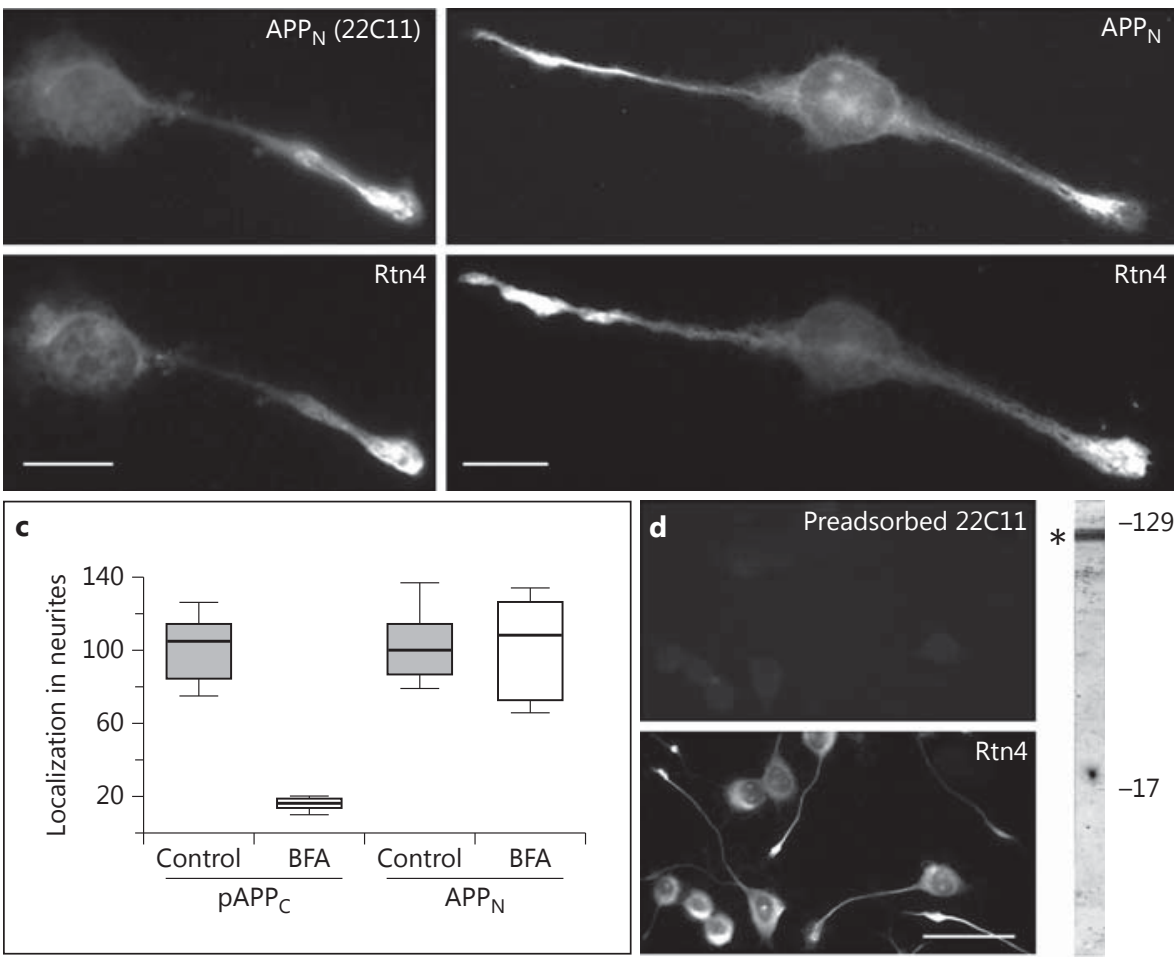

membrane protein specific for the tubular ER [20] (online suppl. fig. 1b). We previously reported that sAPP - endogenous, or derived from exogenously expressed dualtagged APP - localizes to filamentary structures that extend from the soma through the processes, and into the growth cone [16]. Surprisingly, Rtn4-positive, filamentlike structures distribute in a similar pattern along the neurites (online suppl. fig. 1c), suggesting that the trans- ported sAPP could actually be localized within protruding ER tubules. If sAPP was transported into neurites within the ER, this form of transport should not be affected by treatment with BFA, a drug that inhibits ER to Golgi apparatus transport, and thus blocks the transport along the secretory route of conventional, trans-Golgi network (TGN)-derived vesicles [21], but does not affect the extension of the ER. As shown in figure 1a and c, BFA 
Table 1. Similar effect of experimental manipulations that disrupt transport along the secretory route, or the NF network, on the translocation of sAPP and ALS-relevant proteins, in CAD cell neurites

\begin{tabular}{lllllllll}
\hline \multirow{2}{*}{$\begin{array}{l}\text { Experimental } \\
\text { manipulation }\end{array}$} & \multicolumn{7}{l}{ Localization at neurite terminals } \\
\cline { 2 - 7 } & sAPP & CTF pCTF JIP-1 & FUS TDP-43 SOD1 \\
\hline BFA & + & - & - & - & + & + & & + \\
BFA plus washout & + & + & + & + & + & + & & + \\
siRNA peripherin & $\downarrow$ & + & + & + & $\downarrow$ & $\downarrow$ & $\downarrow$ \\
Endogenous condition & + & + & + & + & + & + & \\
\hline
\end{tabular}

Summary of outcome of experiments that test the translocation of $\mathrm{AD}$ - and ALS-relevant proteins into neurites, upon treatment of CAD cells with: BFA, BFA followed by a washout period, or peripherin-specific siRNA. Results were evaluated by comparison with nontreated CAD cells (endogenous condition, bottom row), where all tested proteins showed localization at neurite terminals $(+)$. BFA treatment eliminates the localization of C-terminal APP epitopes (indicative of CTF), Thr ${ }^{668}$-phosphorylated CTF (pCTF), and JIP-1 (a kinesin-1 cargo, transported in association with TGNderived vesicles). Penetration into neurites, and localization at terminals, of sAPP, FUS, TDP-43 and SOD1 is unaffected in BFAtreated cultures, but significantly diminished (downward-pointing arrows) upon transfection with peripherin-specific siRNA.

induces the typical redistribution to the ER of Golgi markers (e.g. GM130), and of cargo proteins such as the CTFs - phosphorylated or not - that normally proceed along the secretory route, and blocks their transport into neurites. Unlike the CTFs, Rtn4- and sAPP-containing structures are unperturbed by BFA treatment, enter the neurites and reach the terminals, as they do in control samples in the absence of BFA (fig. 1b, c). These results are summarized in table 1 and indicate that sAPP is transported into neurites by a nonconventional mechanism that does not implicate TGN-derived secretory/transport vesicles, but which appears to involve extension of ERlike tubules.

We also show that the ALS-relevant, DNA/RNAbinding proteins FUS and TDP-43, while primarily being localized to the nucleus, also display a cytoplasmic distribution pattern reminiscent of that of SAPP and Rtn4 (fig. 2a). This suggests that these proteins could normally be delivered into neurites by associating with the ER. If so, BFA treatment, which blocks only the conventional vesicular transport along the secretory route, should not interfere with the extension of ER tubules in neurites, and with the accumulation of FUS and TDP-43 at terminals. As shown in figure $2 \mathrm{~b}$ and table 1 , the penetration of FUS and TDP-43 into neurites is not blocked in BFA-treated
CAD cells. The clear localization of both proteins to filamentous structures (fig. 2b), rather than the diffuse distribution typically seen for protein complexes free in the cytoplasm, indicates that FUS and TDP-43 are likely carried into the terminals by associating with protruding ER tubules.

About $20 \%$ of the familial cases of ALS are caused by mutations in SOD1 [22]. A recent study with the SOD1 G93A mouse model of ALS indicated that APP contributes to the neuronal pathology [2], but the mechanism remains unresolved. Here, we find that SOD1 largely colocalizes with sAPP in the soma and neurites (fig. 2c); thus, like FUS and TDP-43, SOD1 appears to be transported into neurites attached to Rtn4-positive ER-like extensions. Indeed, SOD1 significantly colocalizes with Rtn4 in the neurites of CAD cells (fig. 2c). As with FUS and TDP-43, the translocation of SOD1 into neurites was not blocked by BFA treatment (table 1). Together, these results are consistent with a transport mechanism where SOD1 associates with the ER protrusions. Thus, within the neurites, SOD1, FUS and TDP-43 behave like peripheral, ER membrane proteins and reach the terminals not by diffusion but by binding to membrane-bound carriers.

The formation of the ER networks is a microtubuledependent process, which relies on microtubule motors [23]. The transport of sAPP also requires the anterograde motor, kinesin-1 [18]. This explains the partial colocalization of sAPP with microtubules - especially the acetylated microtubules - within the neurites, which we previously reported $[13,16]$. However, the extensive colocalization of sAPP with NFs along neurites and at terminals, as we found here, is unexpected. This colocalization is particularly high with peripherin (online suppl. fig. 2a), an NF subunit [24] present in peripheral nervous system and brainstem neurons, and in retinal ganglion cells. Peripherin is required for the normal assembly of the NF network in these neurons [25]. We have previously shown that downregulation of peripherin expression eliminates the filamentary distribution of sAPP in the soma [8]. Here, we report that knocking down peripherin with siRNA in CAD cells - a procedure that decreases peripherin expression by $\sim 60 \%$ and disrupts peripherin-containing NFs within neurites (online suppl. fig. 2b, c) - also diminishes the translocation of sAPP into neurites, and its accumulation at terminals (online suppl. fig. 2b). Likewise, the localization of FUS, TDP-3 and SOD1 at neurite terminals is decreased by $\sim 50 \%$ in CAD cells transfected with peripherin siRNA (data not shown). This is a significant result, since NF proteins have a low turnover and are difficult to completely eliminate with RNA interfer-
58

Neurodegener Dis 2016;16:55-61 DOI: $10.1159 / 000439256$
Muresan/Ladescu Muresan 

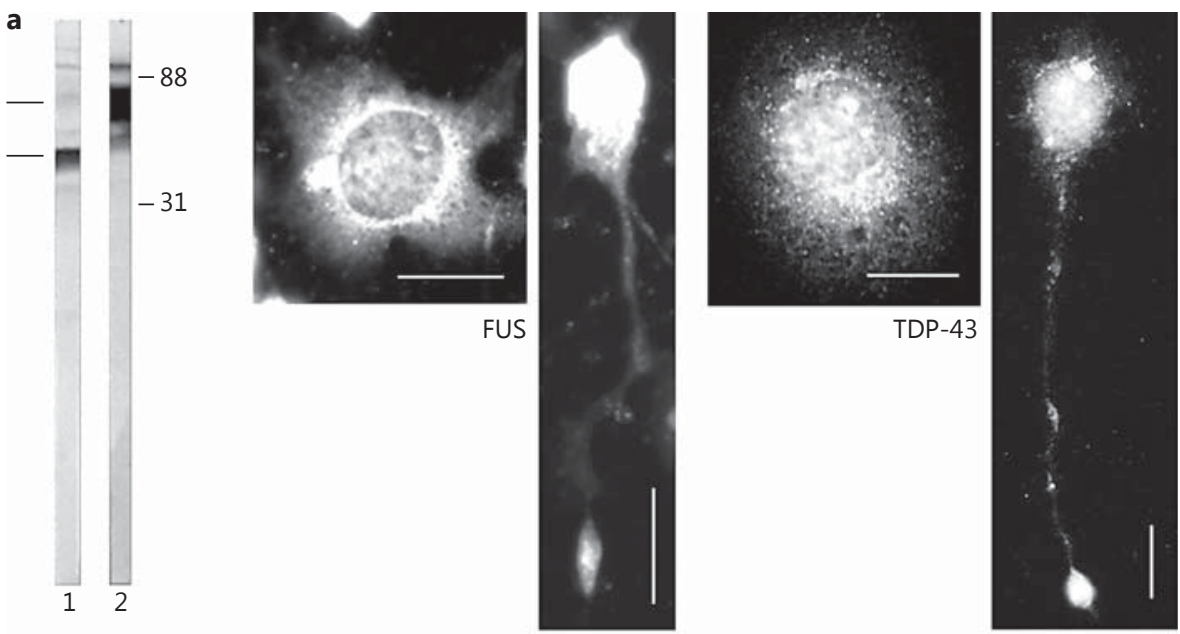

Fig. 2. The distribution patterns of FUS, TDP-43 and SOD1 are similar to those of sAPP and the tubular ER (Rtn4), both in the soma and the neurites, in CAD cells (see also online suppl. fig. 1). a Localization to a broad region in the soma, and accumulation at neurite terminals, of FUS and TDP-43. In cells with flat appearance, the distribution is typical of the ER. The immunoblot shows FUS (lane 2) and TDP-43 (lane 1) in CAD cell extract. Molecular weight markers are given in kilodaltons. b BFA treatment does not affect the localization of FUS and TDP-43 to elongated structures at neurite terminals. The insets show higher magnifications of the terminals (arrows), at increased contrast and brightness. c Colocalization, to the same intracellular structure, of SOD1 with sAPP and $\operatorname{Rtn} 4$, in the soma and at neurite terminals. We note that conventional immunofluorescence microscopy - as used here does not resolve the labeling of membraneassociated proteins, such as SOD1, from that of luminal or transmembrane proteins, such as sAPP and Rtn4. Bars $=20 \mu \mathrm{m}$.

b

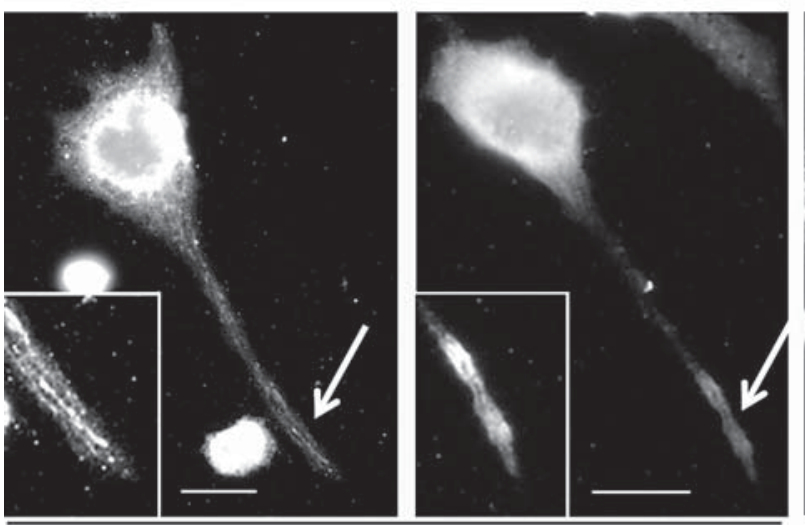

FUS

c

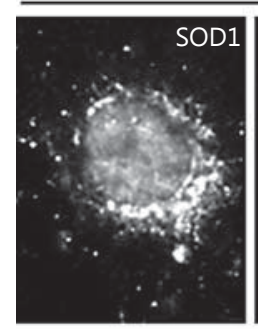

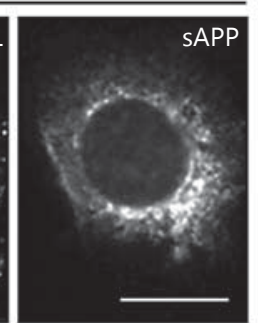

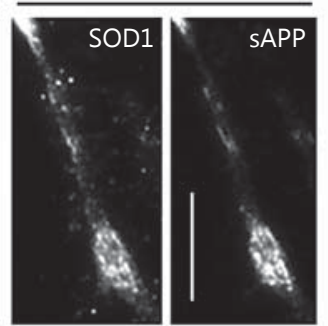

BFA

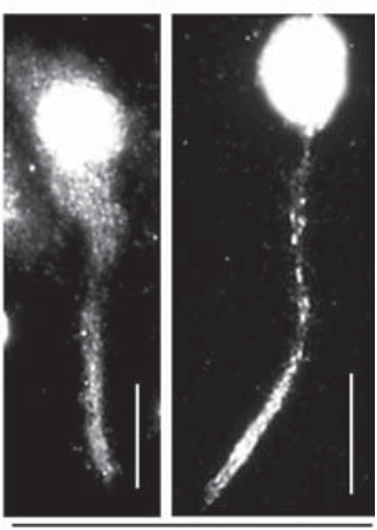

TDP-43

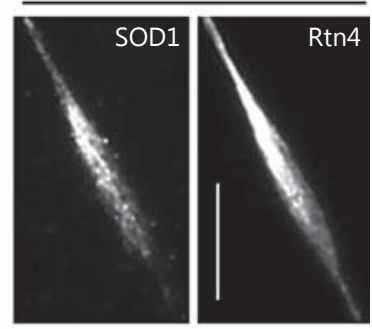

ence. We conclude that NFs are required for the efficient translocation of sAPP, FUS, TDP-43 and SOD1 into the neurites.

\section{Conclusions}

This study identifies a common mechanism of transport into neurites of proteins linked to the pathology of AD (i.e. sAPP) and ALS (i.e. FUS, TDP-43 and SOD1).
This novel form of transport likely relies on Rtn4-containing, ER-derived tubules penetrating into neurites, at long distance [26]. Interestingly, the reticulon protein family, to which Rtn 4 belongs, has been previously linked to both AD [27] and ALS [28]. The study also identifies the NFs - which are disorganized in all cases of ALS - as potential regulators of this mechanism of transport.

Previous studies have shown that a fraction of sAPP, mostly derived by secretase cleavage from the longer isoforms of APP (also known as protease nexin-II), is rap- 
idly secreted into the extracellular space, via TGN-derived vesicles [29]. The reported, extremely fast, time course of secretion of protease nexin-II [29] suggests that this event occurs in the cell body. The TGN-independent transport pathway described by us carries sAPP - likely sAPP $\beta$ [16] - at long distance, to neurite terminals, and certainly occurs in addition to the traditional form of transport. Future studies are required to determine the extent of this novel mechanism of transport, and to precisely identify the sAPP species carried into neurites within ER tubules.

A deficient axonal transport is suspected to be part of the pathogenesis of several neurodegenerative diseases, although this remains to be unequivocally demonstrated [30]. Yet, it is unlikely that gross abnormalities of transport occurring throughout life underlie the pathology of old-age diseases, since no signs of disease are seen until later in life. Rather, more subtle deficiencies that only become manifest over time, or during persistent stress (as occurs in old age), could ultimately lead to disease [31]. More specifically, the disease process likely affects transport of only some specific cargo. Here we tentatively identify a novel form of transport, carrying a defined - yet limited - set of cargo proteins: those that segregate into - or associate with - ER-like tubules extending into neurites, down to the terminal. How the intraluminal sAPP becomes concentrated in the tubular ER-like extensions that protrude into the neurites remains to be determined. Also, how soluble proteins present in the cytoplasm, such as FUS, TDP-43 and SOD1 are tethered to the translocating ER-derived tubules remains to be elucidated. An obvious possibility is that they directly bind to integral, ER membrane proteins present in the protruding tubules. RNA-binding proteins have been previously shown to 'hook' to the ER for transport of vegetal pole- localized RNA in Xenopus oocytes [32, 33]. We note that mitochondria could in principle also carry cytoplasmic proteins into neurites. Our findings do not exclude the possibility that the disease-related proteins investigated here do not also associate to some extent with mitochondria, in addition to the ER, as an alternative mechanism of transport into the neurites.

An interesting finding, to be expanded in future studies, is that NFs likely regulate the type of microtubulebased transport described here. This is a new role of the NFs, which have been consistently viewed as a cytoskeletal network with passive function. Importantly, the NFs appear to regulate the translocation into neurites not only of proteins linked to ALS (a disease where NFs are disorganized), but also of proteins relevant to $\mathrm{AD}$ (a disease with no obvious disruption of the NF cytoskeleton). This points to the necessity to investigate a possible 'malfunction' of the NF network in $\mathrm{AD}$, maybe in a more subtle way than in ALS, yet still causing disease. Indeed, the NFs appear to regulate both the proteolytic cleavage of APP [8] and the intracellular transport of sAPP (this report). We hope that this article will open new and important directions of study in AD and ALS.

\section{Acknowledgments}

This work was supported by National Institutes of Health award AG039668 (to Z.L.M.), National Science Foundation EAGER award IOS-1347090 (to V.M. and Z.L.M.) and New Jersey Health Foundation awards (to Z.L.M. and V.M.).

\section{Disclosure Statement}

The authors have no conflicts of interest to declare.

\section{References}

1 Xiao S, McLean J, Robertson J: Neuronal intermediate filaments and ALS: a new look at an old question. Biochim Biophys Acta 2006; 1762:1001-1012.

- 2 Bryson JB, Hobbs C, Parsons MJ, Bosch KD, Pandraud A, Walsh FS, Doherty P, Greensmith L: Amyloid precursor protein (APP) contributes to pathology in the SOD1(G93A) mouse model of amyotrophic lateral sclerosis. Hum Mol Genet 2012;21:3871-3882.

-3 Caldwell JH, Klevanski M, Saar M, Muller UC: Roles of the amyloid precursor protein family in the peripheral nervous system. Mech Dev 2013;130:433-446.
4 Amador-Ortiz C, Lin WL, Ahmed Z, Personett D, Davies P, Duara R, Graff-Radford NR, Hutton ML, Dickson DW: TDP-43 immunoreactivity in hippocampal sclerosis and Alzheimer's disease. Ann Neurol 2007;61:435445.

${ }_{5} \mathrm{Hu}$ WT, Josephs KA, Knopman DS, Boeve BF, Dickson DW, Petersen RC, Parisi JE: Temporal lobar predominance of TDP-43 neuronal cytoplasmic inclusions in Alzheimer disease. Acta Neuropathol (Berl) 2008; 116:215-220.
6 Murakami K, Murata N, Noda Y, Tahara S, Kaneko T, Kinoshita N, Hatsuta H, Murayama S, Barnham KJ, Irie K, Shirasawa T, Shimizu T: SOD1 (copper/zinc superoxide dismutase) deficiency drives amyloid beta protein oligomerization and memory loss in mouse model of Alzheimer disease. J Biol Chem 2011;286:44557-44568.

7 Spisak K, Klimkowicz-Mrowiec A, Pera J, Dziedzic T, Aleksandra G, Slowik A: rs2070424 of the SOD1 gene is associated with risk of Alzheimer's disease. Neurol Neurochir Pol 2014;48:342-345. 
8 Muresan V, Villegas C, Ladescu Muresan Z: Functional interaction between amyloid-beta precursor protein and peripherin neurofilaments: a shared pathway leading to $\mathrm{Alz}$ heimer's disease and amyotrophic lateral sclerosis? Neurodegener Dis 2014;13:122125.

9 Qi Y, Wang JK, McMillian M, Chikaraishi DM: Characterization of a CNS cell line, $\mathrm{CAD}$, in which morphological differentiation is initiated by serum deprivation. J Neurosci 1997;17:1217-1225.

10 Muresan Z, Muresan V: The amyloid-beta precursor protein is phosphorylated via distinct pathways during differentiation, mitosis, stress, and degeneration. Mol Biol Cell 2007; 18:3835-3844.

11 Muresan Z, Muresan V: Seeding neuritic plaques from the distance: a possible role for brainstem neurons in the development of Alzheimer's disease pathology. Neurodegener Dis 2008;5:250-253.

12 Muresan Z, Muresan V: CAD cells are a useful model for studies of APP cell biology and Alzheimer's disease pathology, including accumulation of $A \beta$ within neurites. Swan Alzheimer knowledge base. Alzheimer Research Forum. 2009. http://mind-swanweb1.Mgh. Harvard.Edu/swan/browser/showentity.Acti on?Objectid=urn\%3alsid\%3aswan.Org\%3are searchstatement\%3aca7169f1-ff7d-456cb3f3-c52bec1e8074.

13 Muresan V, Varvel NH, Lamb BT, Muresan $\mathrm{Z}$ : The cleavage products of amyloid-beta precursor protein are sorted to distinct carrier vesicles that are independently transported within neurites. J Neurosci 2009;29:35653578.

14 Muresan Z, Muresan V: A phosphorylated, carboxy-terminal fragment of beta-amyloid precursor protein localizes to the splicing factor compartment. Hum Mol Genet 2004;13: 475-488.

$\checkmark 15$ Muresan Z, Muresan V: Neuritic deposits of amyloid- $\beta$ peptide in a subpopulation of cen- tral nervous system-derived neuronal cells. Mol Cell Biol 2006;26:4982-4997.

16 Villegas C, Muresan V, Ladescu Muresan Z: Dual-tagged amyloid-beta precursor protein reveals distinct transport pathways of its $\mathrm{N}$ and C-terminal fragments. Hum Mol Genet 2014;23:1631-1643.

17 Muresan Z, Muresan V: c-Jun NH2-terminal kinase-interacting protein-3 facilitates phosphorylation and controls localization of amyloid-beta precursor protein. J Neurosci 2005; 25:3741-3751.

18 Muresan Z, Muresan V: Coordinated transport of phosphorylated amyloid-beta precursor protein and c-Jun NH2-terminal kinaseinteracting protein-1. J Cell Biol 2005;171: 615-625.

19 Spitzer M, Wildenhain J, Rappsilber J, Tyers M: Boxplotr: a web tool for generation of box plots. Nat Methods 2014;11:121-122.

20 Voeltz GK, Prinz WA, Shibata Y, Rist JM, Rapoport TA: A class of membrane proteins shaping the tubular endoplasmic reticulum. Cell 2006;124:573-586.

21 Lippincott-Schwartz J, Donaldson JG, Schweizer A, Berger EG, Hauri HP, Yuan LC, Klausner RD: Microtubule-dependent retrograde transport of proteins into the ER in the presence of brefeldin A suggests an ER recycling pathway. Cell 1990;60:821-836.

22 Rosen DR, Siddique T, Patterson D, Figlewicz DA, Sapp P, Hentati A, Donaldson D, Goto J, O’Regan JP, Deng HX, et al: Mutations in Cu/ $\mathrm{Zn}$ superoxide dismutase gene are associated with familial amyotrophic lateral sclerosis. Nature 1993;362:59-62.

23 Wozniak MJ, Bola B, Brownhill K, Yang YC, Levakova V, Allan VJ: Role of kinesin-1 and cytoplasmic dynein in endoplasmic reticulum movement in Vero cells. J Cell Sci 2009;122: 1979-1989.

24 Escurat M, Djabali K, Gumpel M, Gros F, Portier MM: Differential expression of two neuronal intermediate-filament proteins, peripherin and the low-molecular-mass neuro- filament protein (NF-1), during the development of the rat. J Neurosci 1990;10:764-784.

25 Yuan A, Sasaki T, Kumar A, Peterhoff CM, Rao MV, Liem RK, Julien JP, Nixon RA: Peripherin is a subunit of peripheral nerve neurofilaments: implications for differential vulnerability of CNS and peripheral nervous system axons. J Neurosci 2012;32:8501-8508.

26 Muresan V, Ladescu Muresan Z: Amyloidbeta precursor protein: multiple fragments, numerous transport routes and mechanisms. Exp Cell Res 2015;334:45-53.

$27 \mathrm{He}$ W, Lu Y, Qahwash I, Hu XY, Chang A, Yan R: Reticulon family members modulate BACE1 activity and amyloid-beta peptide generation. Nat Med 2004;10:959-965.

28 Yang YS, Harel NY, Strittmatter SM: Reticulon-4A (Nogo-A) redistributes protein disulfide isomerase to protect mice from SOD1dependent amyotrophic lateral sclerosis. J Neurosci 2009;29:13850-13859.

-29 Sambamurti K, Shioi J, Anderson JP, Pappolla MA, Robakis NK: Evidence for intracellular cleavage of the Alzheimer's amyloid precursor in PC12 cells. J Neurosci Res 1992;33:319329 .

30 Muresan V, Muresan Z: Is abnormal axonal transport a cause, a contributing factor or a consequence of the neuronal pathology in Alzheimer's disease? Future Neurol 2009;4: 761-773.

31 Muresan V, Muresan Z: A persistent stress response to impeded axonal transport leads to accumulation of amyloid-beta in the endoplasmic reticulum, and is a probable cause of sporadic Alzheimer's disease. Neurodegener Dis 2012;10:60-63.

32 Deshler JO, Highett MI, Abramson T, Schnapp BJ: A highly conserved RNA-binding protein for cytoplasmic mRNA localization in vertebrates. Curr Biol 1998;8:489-496.

33 Deshler JO, Highett MI, Schnapp BJ: Localization of Xenopus Vg1 mRNA by Vera protein and the endoplasmic reticulum. Science 1997;276:1128-1131. 